John P. Perdew*, Jianwei Sun, Alejandro J. Garza, and

Gustavo E. Scuseria

\title{
Intensive Atomization Energy: Re-Thinking a Metric for Electronic Structure Theory Methods
}

DOI 10.1515/zpch-2015-0713

Received October 15, 2015; accepted December 14, 2015

Abstract: The errors in atomization energies $(A E)$ of molecules have long been used to measure the errors of wavefunction or density functional methods for electronic structure calculations. In particular, the G3 set of Pople and collaborators (for $s p$-bonded molecules from the first rows of the periodic table) has become a standard benchmark for such methods. But the mean absolute error of $A E$ tends to increase with increasing number $N_{\text {at }}$ of atoms in a molecule. In fact, $A E$ is an extensive variable, which diverges as $N_{\text {at }} \rightarrow \infty$. Here, as did Savin and Johnson 2015, we define an intensive atomization energy, $I A E=A E / N_{\text {at }}$ or atomization energy per atom, which tends to the finite cohesive energy (per atom) of a large cluster or solid $\left(N_{\text {at }} \rightarrow \infty\right)$. We find that the mean absolute error of the G3 molecular IAE from accurate density functionals remains close to $1 \mathrm{kcal} / \mathrm{mol}$ as the average molecular size increases. This makes it possible to estimate in advance the magnitude of the error in $A E$ for a molecule similar to most of those in the G3 set. It also allows us identify the G3 "outlying molecules", and to more directly compare the accuracy of a given functional for different kinds of molecules (such as those containing transition-metal atoms) to that for G3-type molecules, by removing the otherwise-uncontrolled size factor. Finally, we point out that the familiar concept of "chemical accuracy" needs to be qualified.

Keywords: DFT, Atomization Energies, Intensive Variables, Extensive Variables, Standard Benchmark, Chemical Accuracy.

*Corresponding author: John P. Perdew, Physics Department, Temple University, Philadelphia, PA 19122, USA; and Chemistry Department, Temple University, Philadelphia, PA 19122, USA, e-mail: tuf25956@temple.edu

Jianwei Sun: Physics Department, Temple University, Philadelphia, PA 19122, USA

Alejandro J. Garza: Department of Chemistry, Rice University, Houston, TX 77251, USA Gustavo E. Scuseria: Department of Chemistry, Rice University, Houston, TX 77251, USA; and Department of Physics and Astronomy, Rice University, Houston, TX 77251, USA; and Chemistry Department, Faculty of Science, King Abdulaziz University, Jeddah, Saudi Arabia 
Dedicated to Professor Michael Springborg on the occasion of his $60^{\text {th }}$ birthday

The atomization energy $A E$ of a molecule is the minimum energy needed to break up the molecule into well-separated atoms. The error statistics for calculated atomization energies have played an important role as a metric for electronic structure methods. This is a convenient choice for correlated wavefunction methods, which can be applied to atoms and molecules but not so easily to solids. The improvement over the local spin-density approximation for atomization energies, achieved by generalized gradient approximations and hybrid functionals in the 1990s, was an important historical factor leading to the wide acceptance of KohnSham density functional theory [1] by chemists, and to the 1998 Nobel Prize in Chemistry shared by Walter Kohn and John Pople. Density functional theory is of course as useful in condensed matter physics and materials science as it is in chemistry.

This is the third of a series of papers some of us have written about atomization energies. Reference [2] pointed out that, from our current perspective, "density functionals should not be judged primarily by atomization energies" [3-6]. The atoms are open-shell and typically spin-polarized, while the molecules or solids are more typically closed-shell and spin-unpolarized. Thus errors of calculated atomization energies can arise from errors in the spin-polarization dependence of the density functional that are much less relevant to chemical reaction energies including formation energies. Reference [7] pointed out that, while atomization energies in spin-density functional theory improve dramatically with the degree of constraint satisfaction of the tested semilocal functional, this is not the case in total-density functional theory, where the energies of spin-polarized systems are not amenable to semilocal approximation because the on-top exchangecorrelation holes are only accurate in the spin-density functionals [8].

Here we want to address a different problem with atomization energies as a metric for electronic structure methods: They are extensive, increasing with the number $N_{\text {at }}$ of atoms in the molecule, and their errors are similarly extensive. This can be seen in Table 1 [9], for the three subsets of the standard G3 set of molecules proposed by Curtiss, Raghavachari, Redfern, and Pople [10]. These are all $s p$-bonded molecules from the first rows of the periodic table. The G3 set was compiled to test the G3 or Gaussian3 quantum chemical method. The G3 subsets are not systematically organized, but there is a clear increase in the average size of a molecule from G3-1 to G3-2 to G3-3. Each subset is large enough to provide meaningful error statistics. For a given functional, the G3-1 set of 55 small molecules has a smaller mean absolute error than the G3-2 set of 93 larger molecules, and that in turn has a smaller mean absolute error than the G3-3 set of 75 mostly-large 
Table 1: Mean absolute error in $\mathrm{kcal} / \mathrm{mol}$ of atomization energy for the three subsets and for the full G3 set [9]. The average number of atoms in a molecule increases from G3-1 to G3-2 to G3-3. The number of molecules in a set is indicated in parentheses after the set.

\begin{tabular}{lrrrr}
\hline Functional & G3-1(55) & G3-2(93) & G3-3(75) & G3(223) \\
\hline Hartree-Fock & 75.4 & 192.9 & 337.3 & 212.5 \\
LSDA & 36.3 & 111.1 & 196.6 & 121.4 \\
BLYP GGA & 4.8 & 8.7 & 13.9 & 9.5 \\
PBE GGA & 8.2 & 22.0 & 32.8 & 22.2 \\
M06L meta-GGA & 3.5 & 5.2 & 6.4 & 5.2 \\
SCAN meta-GGA & 3.3 & 5.3 & 8.0 & 5.7 \\
\hline
\end{tabular}

organic molecules. We have included the Hartree-Fock (HF) method, the nonempirical local spin density approximation (LSDA) [11, 12], two generalized gradient approximations (GGAs) [13-15], and two meta-GGAs [16, 17]. Note that HF, which is a wavefunction method, shows the same trends as the density functionals (albeit the errors are much larger). The PBE GGA [15] and the SCAN meta-GGA [17] were constructed nonempirically by satisfaction of known exact constraints on the density functional for the exchange-correlation energy. SCAN satisfies all 17 known exact constraints that a semilocal functional can. All calculations have been made using the Gaussian code [18] with the $6-311++\mathrm{G}(3 d f, 3 p d)$ basis set and the pruned $(99,590)$ grid (ultrafine).

We can construct an intensive atomization energy

$$
I A E=A E / N_{\text {at }},
$$

or atomization energy per atom, which tends to the cohesive energy (per atom) of a large cluster or solid as $N_{\text {at }} \rightarrow \infty$. For the G3-1 and G3-2 subsets together, the mean experimental intensive atomization energy is $80 \mathrm{kcal} / \mathrm{mol}$ from Table 1 of Reference [19]. Table 2 shows the mean absolute errors of intensive atomization energy. They are about the same for all three subsets of G3, despite the increase in $\left\langle N_{\text {at }}\right\rangle$ from 3 (G3-1) to 7 (G3-2) to 12 (G3-3). For the most-accurate semilocal functionals (the meta-GGAs), this error is about $1 \mathrm{kcal} / \mathrm{mol}=0.04 \mathrm{eV}$.

In fact, for a given functional Table 2 shows a tendency for the mean absolute error of $I A E$ to decrease slightly from G3-1 to G3-2 to G3-3. This may reflect the presence of many small "outlying molecules" in G3-1, and the increasing presence in the other sets of large hydrocarbons, which may be especially amenable to semilocal approximation [17].

Is the IAE idea useful? First, it can be used to identify the true outliers among G3 and G3-like molecules, as in Table 3. Second, it could be used to estimate the magnitude of the atomization energy error $\propto N_{\text {at }}$ for a given functional applied to 
Table 2: Mean absolute error in $\mathrm{kcal} / \mathrm{mol}$ of intensive atomization energy of Equation (1) for the three subsets and for the full $\mathrm{G} 3$ set. The number of molecules in a set is indicated in parentheses after the set.

\begin{tabular}{lrrrr}
\hline Functional & G3-1(55) & G3-2(93) & G3-3(75) & G3(223) \\
\hline Hartree-Fock & 25.1 & 28.3 & 27.6 & 27.3 \\
LSDA & 12.0 & 16.4 & 16.3 & 15.3 \\
BLYP GGA & 1.9 & 1.6 & 1.1 & 1.5 \\
PBE GGA & 3.1 & 3.7 & 3.0 & 3.3 \\
M06L meta-GGA & 1.3 & 0.9 & 0.7 & 0.9 \\
SCAN meta-GGA & 1.3 & 1.0 & 0.8 & 1.0 \\
\hline
\end{tabular}

Table 3: G3 outlier molecules for the SCAN meta-GGA, their signed errors in $\mathrm{kcal} / \mathrm{mol}$ for intensive atomization energy, and the subsets to which they belong.

\begin{tabular}{lrrlrr}
\hline Molecule & Error & Subset & Molecule & Error & Subset \\
\hline $\mathrm{BeH}$ & 5.3 & $\mathrm{G} 3-1$ & $\mathrm{CS}_{2}$ & 2.9 & $\mathrm{G} 3-2$ \\
$\mathrm{~N}_{2}$ & -4.9 & $\mathrm{G} 3-1$ & $\mathrm{PCl}_{5}$ & 2.8 & $\mathrm{G} 3-3$ \\
$\mathrm{O}_{2}$ & 3.6 & $\mathrm{G} 3-1$ & $\mathrm{SiO}$ & -2.8 & $\mathrm{G} 3-1$ \\
$\mathrm{~S}_{2}$ & 3.5 & $\mathrm{G} 3-1$ & $\mathrm{~F}_{2} \mathrm{C}=\mathrm{CF}_{2}$ & 2.8 & $\mathrm{G} 3-2$ \\
$\mathrm{ClF}_{3}$ & 3.3 & $\mathrm{G} 3-2$ & $\mathrm{COS}$ & -2.6 & $\mathrm{G} 3-2$ \\
$\mathrm{BCl}_{3}$ & 3.3 & $\mathrm{G} 3-2$ & $\mathrm{SiH}_{2}\left({ }^{3} \mathrm{~B}_{1}\right)$ & 2.5 & $\mathrm{G} 3-1$ \\
$\mathrm{SO}$ & 3.2 & $\mathrm{G} 3-1$ & $\mathrm{ClO}$ & 2.5 & $\mathrm{G} 3-1$ \\
$\mathrm{CF}_{3}$ & 3.1 & $\mathrm{G} 3-3$ & $\mathrm{AlCl}_{3}$ & 2.5 & $\mathrm{G} 3-2$ \\
$\mathrm{Cl}_{2} \mathrm{~S}_{2}$ & 3.0 & $\mathrm{G} 3-3$ & $\mathrm{C}_{2} \mathrm{Cl}_{4}$ & 2.2 & $\mathrm{G} 3-2$ \\
$\mathrm{Li}_{2}$ & -3.0 & $\mathrm{G} 3-1$ & & & \\
\hline
\end{tabular}

an expected non-outlier. Third, it could be used to make a more meaningful comparison between the error of a given functional for G3-like and non-G3-like (e.g., transition-metal-containing) molecules, by removing the size effect from the comparison. Non-G3-like molecules are built up from atoms having $d$ or $f$ electrons, or large relativistic effects.

Table 3 identifies 19 G3 IAE outliers for the SCAN meta-GGA. All are relatively small molecules. Many contain $\mathrm{Si}, \mathrm{S}$, or $\mathrm{Cl}$, and some contain $\mathrm{O}$ and $\mathrm{F}$. (Yet $\mathrm{H}_{2} \mathrm{O}$, with an error of $-0.9 \mathrm{kcal} / \mathrm{mol}$, is quite normal, as is $\mathrm{SF}_{6}$, with an error of $1.5 \mathrm{kcal} / \mathrm{mol}$.) A few molecules $\left(\mathrm{BeH}, \mathrm{N}_{2}\right.$, and $\mathrm{Li}_{2}$ ) achieve large errors without containing any of those elements. Probably radicals like $\mathrm{BeH}, \mathrm{O}_{2}, \mathrm{~S}_{2}$, and $\mathrm{SO}$ require a self-interaction correction. Note also that the largest error occurs for $\mathrm{BeH}$ - the only molecule in the G3 set containing beryllium - and that there is static correlation in the Be atom which is not well described by commonly used Kohn-Sham semilocal functionals. (The error in the total energy of the Be atom 
for SCAN is about $10.9 \mathrm{kcal} / \mathrm{mol}$ [20].) Further analysis is needed to understand the outliers.

Our intensive atomization energy is different from the atomization energy per bond defined by Zhang, Truhlar and Tang [21], with for example a double bond counted as one bond. The latter quantity is however also intensive, because the number of bonds per atom saturates as the number of atoms in a molecule or cluster tends to infinity. We prefer our definition, which successfully reduces the size-dependence of the mean absolute error of the atomization energy and does not require counting bonds. Bonds are less well-defined than atoms.

A familiar concept is "chemical accuracy", meaning typical errors on the order of $1 \mathrm{kcal} / \mathrm{mol}$. This concept however needs qualification. If it means a $1 \mathrm{kcal} / \mathrm{mol}$ error in an intensive quantity like $I A E$, then the nonempirical SCAN meta-GGA already achieves it. If it means a $1 \mathrm{kcal} / \mathrm{mol}$ error in an extensive quantity like $A E$, then it is only meaningful for particular data sets like G3. If we expand those data sets to include larger and larger molecules, then the mean absolute error for any electronic-structure-theory method will diverge with the average number of atoms per molecule. Polymers [22], which can be extended periodically and indefinitely, are a compelling paradigm.

After this work was submitted, a referee pointed out the recent work of Savin and Johnson 2015 [23], which also defines an atomization energy per atom and shows, for a different set of functionals including hybrid functionals, that the corresponding mean absolute error is approximately independent of molecular size over the G3-1, G3-2, and G3-3 subsets (reaching about $1 \mathrm{kcal} / \mathrm{mol}$ for standard hybrids). Reference [23] presents a broader discussion of "Judging Density Functional Approximations: Some Pitfalls of Statistics”. We believe that our article still has value, for several reasons: (1) Its title and abstract call attention to intensive atomization energy or atomization energy per atom, which are not mentioned in the title, abstract, and introduction of Reference [23]. (2) We test density functionals, including meta-GGAs, that are not tested in Reference [23]. We also stress the testing of nonempirical methods like Hartree-Fock, LSDA, PBE GGA, and the recent SCAN meta-GGA. (3) We suggest several uses for the intensive atomization energy, as summarized in our abstract, that are not addressed in Reference [23].

Acknowledgement: This work is primarily supported as part of the Center for the Computational Design of Functional Layered Materials, an Energy Frontier Research Center funded by the U.S. Department of Energy, Office of Basic Energy Sciences, under Award \# DE-SC0012575 (computational aspects), and by the National Science Foundation under grant DMR-1305135 (design of the project). GES is a Welch Foundation Chair (C-0036). JPP is a Laura H. Carnell Professor. JPP has benefitted from discussions of polymers with Michael Springborg. 


\section{References}

1. W. Kohn and L. J. Sham, Phys. Rev. 140 (1965) A1133.

2. J. P. Perdew, J. Sun, A. Ruzsinszky, P. D. Mezei, and G. I. Csonka, Period. Polytech. Chem. DOI:10.3311/PPCh.8356 (2015).

3. B. Delley, J. Chem. Phys. 113 (2000) 7756.

4. G. I. Csonka, A. Ruzsinszky, J. Tao, and J. P. Perdew, Int. J. Quantum Chem. 101 (2005) 506.

5. S. Grimme, J. Phys. Chem. A 109 (2005) 3067.

6. B. Delley, J. Phys. Chem. A 110 (2006) 13632.

7. A. J. Garza, G. E. Scuseria, A. Ruzsinszky, J. Sun, and J. P. Perdew, Mol. Phys. DOI:10.1080/00268976.2015.1114165 (2015).

8. K. Burke, J. P. Perdew, and M. Ernzerhof, J. Chem. Phys. 109 (1998) 3760.

9. Original data are from References $[7,17]$ and used here for a different purpose.

10. L. A. Curtiss, K. Raghavachari, P. C. Redfern, and J. A. Pople, J. Chem. Phys. 112 (2000) 7374.

11. U. von Barth and L. Hedin, J. Phys. C 5 (1972) 1629.

12. J. P. Perdew and Y. Wang, Phys. Rev. B 45 (1992) 13244.

13. A. D. Becke, Phys. Rev. A, 38 (1988) 3098.

14. C. Lee, W. Yang, and R. G. Parr, Phys. Rev. B 37 (1988) 785.

15. J. P. Perdew, K. Burke, and M. Ernzerhof, Phys. Rev. Lett. 77 (1996) 3865.

16. Y. Zhao and D. G. Truhlar, J. Chem. Phys. 125 (2006) 194101.

17. J. Sun, A. Ruzsinszky, and J. P. Perdew, Phys. Rev. Lett. 115 (2015) 036402.

18. M. J. Frisch, G. W. Trucks, H. B. Schlegel, et al., Gaussian 09, Gaussian, Inc., Wallingford, CT (2009).

19. M. Ernzerhof and G. E. Scuseria, J. Chem. Phys. 110 (1999) 5032.

20. A. J. Garza, I. W. Bulik, A. G. Sousa Alencar, J. Sun, J. P. Perdew, and G. E. Scuseria, Mol. Phys. DOI:10.1080/00268976.2015.1114165 (2015); arXiv:1509.03251 [physics.chem-ph].

21. W. Zhang, D. G. Truhlar, and M. Tang, J. Chem. Theory Comput. 9 (2013) 3965.

22. J. Vargas, M. Springborg, and M. Kirtman, J. Chem. Phys. 140 (2014) 054117.

23. A. Savin and E. R. Johnson, Top. Curr. Chem. 365 (2015) 81. 